\title{
THE CONVERGENCE OF $\alpha$ SCHEMES FOR CONSERVATION LAWS I: SEMI-DISCRETE CASE*
}

\author{
NAN JIANG ${ }^{\dagger}$
}

\begin{abstract}
An orderly procedure of constructing families of $\alpha$ and $\beta$ schemes, to approximate homogeneous conservation laws, was introduced by S. Osher and S. Chakravarthy [1,2]. Among these schemes, we are interested in the entropy consistence of the semi-discrete $\alpha$ schemes in the context of approximating scalar conservation laws. In general $\alpha$ schemes, for $0<\alpha \leq \frac{1}{2}$ and $m=2,3 \cdots, 8$, are $2 m-2$ order accurate methods. However when $\alpha=\frac{2}{m}\left(\begin{array}{c}2 m \\ m\end{array}\right)^{-1}$, we obtain one order higher, i.e. $2 m-1$ order, accurate schemes. Although, for the homogeneous conservation laws, Osher and Chakravarthy were able to show the total variation diminishing (TVD) property of these schemes, the entropy convergence of the schemes has been open. In this paper, for all admissible values of $\alpha$ and $m=2$, we extend $\alpha$ schemes to approximate the non-homogeneous convex conservation laws, which in general are total variation bounded (TVB). Finally, using one of our convergence criteria [22], we have established the entropy convergence for both the original and extended $\alpha$ schemes.
\end{abstract}

Key words. Conservation law with source terms, $\alpha$ schemes, entropy condition, convergence.

AMS subject classifications. 65M12 (35L60).

1. Introduction. A reasonably large class of high order schemes for homogeneous hyperbolic conservation laws was developed by Osher and Chakravarthy in [1], where they presented a systematic procedure of constructing general $\alpha$ and $\beta$ conservative schemes. For $m=2,3, \cdots, 8$, these schemes are families of $2 m-2$ and $2 m-1$ order accuracy respectively. In order to attain higher order accurate schemes, they have used different construction ideas than the ones given by Sweby [16]. Osher and Chakravarthy have also successfully established the TVD property of these schemes. The computational aspects of the schemes have been emphasized in their parallel work [2], where Osher and Chakravarthy have demonstrated the effectiveness of the $\alpha$ schemes by applying them to Euler equations of compressible inviscid flows and scalar equations. The numerical results have revealed that these methods offer substantial improvements in the accuracy of numerical solutions and the reliability when they are compared to the earlier generation of second order accurate TVD schemes. This kind of performance should not render a surprise to us at all as the design principle of the schemes has been closely followed the physics. The methods are upwind biased, TVD and utilizing $E$-schemes as building blocks, all of which enhance the schemes with the ability to avoid unphysical "wiggles" and expansion shocks in the computational domain. In this paper, we aim to provide theoretical confirmation to the phenomenal performance of these methods by showing the important entropy convergence of the $\alpha$ schemes. We also prove the entropy consistence of their natural extension to approximate hyperbolic conservation laws with source terms. For the non-homogeneous problems, TVD property of the methods in general will no longer hold, nevertheless the TVB property of such schemes do follow from our previous result in [22].

Let $B V$ stand for the subspace of $L_{l o c}^{1}$ consisting of functions $z$ with bounded total variation

$$
T V(z):=\sup _{h \neq 0} \int_{\mathbb{R}} \frac{|z(x+h)-z(x)|}{|h|} d x
$$

\footnotetext{
*Received June 8, 2012; accepted for publication January 15, 2013.

$\dagger$ Department of Mathematical Sciences, University of South Dakota, Vermillion SD 57069, USA (njiang@usd.edu).
} 
We consider the initial value problems of hyperbolic conservation laws with source terms:

$$
\left\{\begin{array}{l}
w_{t}+f(w)_{x}=q(w), \\
w(x, 0)=w_{0}(x),
\end{array}\right.
$$

where $f \in C^{1}(\mathbb{R})$ is convex, $q \in C^{1}(\mathbb{R})$, and $w_{0} \in B V(\mathbb{R})$. The corresponding homogeneous problems of (1.1) are

$$
\left\{\begin{array}{l}
w_{t}+f(w)_{x}=0 \\
w(x, 0)=w_{0}(x)
\end{array}\right.
$$

It is well known that the solution of (1.1) may develop discontinuities in finite time. Therefore, we seek weak solutions $w$ that satisfies:

$$
\int_{\mathbb{R}} \int_{0}^{T}\left(w \phi_{t}+f(w) \phi_{x}+q(w) \phi\right) d x d t=-\int_{\mathbb{R}} w_{0}(x) \phi(x, 0) d x
$$

for all $\phi \in C_{0}^{1}(\mathbb{R} \times[0, T))$. The week solutions are not necessarily unique and the entropy conditions are required to single out the physically relevant one, which, for all convex entropy function $V$ and its flux $F$, i.e. $V^{\prime}(w) f^{\prime}(w)=F^{\prime}(w)$, satisfies the inequality $[10,19]$

$$
V(w)_{t}+F(w)_{x} \leq V^{\prime}(w) q(w),
$$

in the sense of distribution. Since $f$ is convex, the entropy inequality (1.4) for the entropy function $V(w)=w^{2}$ is sufficient to ensure the uniqueness of the solution [4].

The semi-discrete schemes that we consider have the form

$$
\frac{d u_{j}(t)}{d t}=-\frac{1}{h}\left[g_{j+\frac{1}{2}}(t)-g_{j-\frac{1}{2}}(t)\right]+q\left(u_{j}(t)\right),
$$

with the numerical flux $g$ given by

$$
g_{j+\frac{1}{2}}(t)=g\left(u_{j-p+1}(t), u_{j-p+2}(t), \cdots, u_{j}(t), \cdots, u_{j+p}(t)\right) .
$$

The flux $g_{j+\frac{1}{2}}(t)$ also satisfies the consistent property $g(u, u, \cdots, u) \equiv f(u)$, and is Lipschitz continuous with respect to its $2 p$ arguments.

The homogeneous counterpart of (1.5)-(1.6) that approximate problems (1.2) has the form

$$
\frac{d u_{j}(t)}{d t}=-\frac{1}{h}\left[g_{j+\frac{1}{2}}(t)-g_{j-\frac{1}{2}}(t)\right] .
$$

Traditionally, we approach the entropy convergence analysis of the schemes (1.5)(1.6) by using the classical cell entropy inequality (CEI) method, which demands to establish entropy inequality for each computational cell. Consequently, the entropy convergence of many extremely well-performed schemes, such as $\alpha$ and $\beta$ schemes, has been open. Recent years, various approaches have been developed. Among them, we especially appreciate Yang's wavewise entropy inequality (WEI) [20, 21] concept, due to the fact that it has been shown to be very effective in the convergence analysis. For scalar convex conservation laws, this new concept has produced four convergence criteria for general semi-discrete and fully-discrete schemes, respectively. One of criteria, in semi- or fully-discrete case, essentially states that, a WEI across the area of 
rarefaction where $u_{j} \leq u_{j+1}$ for all $x_{j}$ is sufficient for the convergence to the entropy solution. Hence, in the convergence analysis, one may safely remove the shock area from scrutiny. Further, even in the rarefaction area, a much weaker condition than CEI is sufficient for the convergence. To demonstrate that this approach has brought a powerful tool into the success of convergence analysis, we would like to mention some of our convergence results $[7,8,9,20,21,22]$ in order.

In the semi-discrete case, for the scalar homogeneous convex conservation laws, Yang [20] has shown the entropy convergence of the generalized MUSCL scheme and the schemes based on minmod limiter [15] when the general building block of the schemes is an arbitrary $E$-scheme [14], and based on Chakravarthy-Osher limiter [3] when the building block of the schemes is the Godunov [6], the Engquist-Osher [5], or the Lax-Friedrichs [12]. Few years ago, Yang and the author [7, 22] had made some noticeable advances at this front. We have extended Yang's WEI frame work and convergence results to the convex conservation laws with an arbitrary $C^{1}$ source term. Furthermore, we have established that, for convex conservation laws with or without a source term, the schemes with van Leer's limiter $[16,18]$, when the building block of the schemes is the Godunov, the Engquist-Osher, or the Lax-Friedrichs, converge to the physically correct solution as well.

In the fully-discrete case, for the scalar homogeneous convex conservation laws, Yang [21] has shown the entropy convergence of explicit MUSCL scheme. Recently, the author [8] has shown the entropy convergence of the schemes with van Leer's limiter when the building block of the schemes is the Godunov or the Engquist-Osher.

In this paper, our goal is to use the WEI convergence criterion, formulated in [22], to show the entropy consistency of the $\alpha$ schemes when the building block of the schemes is the Lax-Friedrichs scheme, or a subclass of $E$-schemes of which the Godunov and the Engquist-Osher schemes are special cases. The results obtained here will be valid for scalar convex conservation laws with or without a source term. The same issue of the $\beta$ schemes has been addressed in a companion paper by the author. The paper is organized as follows. In section 2 , we present the main result of this paper. In section 3 , we give the proof of the main result.

2. The main result. For the purpose of self-containment, we state the WEI convergence criterion in this section, which was first established in [20] for homogeneous conservation laws and was extended in [22] for conservation laws with an arbitrary $C^{1}$ source term. It is starting that the WEI given by (2.1) in Theorem 2.3 is the same for the schemes (1.5)-(1.6) and (1.6)-(1.7) that approximate non-homogeneous and homogeneous conservation laws, respectively. Indeed, the source term does not appear in the WEI (2.1). Therefore, the entropy convergence of all schemes established by Theorem 2.3 will remain true with or without a source term.

First, we recall the following separation property given by Assumption 2.1. It is a sufficient condition for the schemes (1.5)-(1.6) to be TVB [22]. This property also services as a convenient TVD condition for the schemes (1.6)-(1.7), which was the result of Tadmor [17].

Assumption 2.1. The numerical fluxes $g_{j+\frac{1}{2}}(t), j=0, \pm 1, \pm 2, \cdots$, satisfy

$$
g_{j+\frac{1}{2}}(t) \geq f\left(u_{j}\right) \geq g_{j-\frac{1}{2}}(t) \quad \text { if } u_{j}(t)-u_{j \pm 1}(t) \geq 0,
$$

and

$$
g_{j+\frac{1}{2}}(t) \leq f\left(u_{j}\right) \leq g_{j-\frac{1}{2}}(t) \quad \text { if } \quad u_{j}(t)-u_{j \pm 1}(t) \leq 0 .
$$


As a result of [22], schemes satisfied the above assumption are extremum traceable. Therefore the WEI convergence criteria are applicable. Let $f[w ; L, R]$ be the linear function interpolating of $f(w)$ at $w=L$ and $w=R$. We define a rarefying pair and a rarefying collection with respect to the pair as follows.

Definition 2.2. For a convex conservation law, a pair of numbers $\{L, R\}$ is called a rarefying pair if $L<R$ and $f[w ; L, R]>f(w)$ when $L<w<R$. A collection of data $\left\{v_{j}\right\}_{j=-p}^{n+p}$ is called a rarefying collection with respect to the pair $\{L, R\}$ if $L=v_{0} \leq v_{1} \leq \cdots \leq v_{n}=R, L \leq v_{-1}$, and $R \geq v_{n+1}$.

Let $\bar{g}_{j+1 / 2}:=g\left(v_{j-p+1}, v_{j-p+2}, \cdots, v_{j+p}\right)$, where $g$ is the function defined by (1.6) that evaluated at a rarefying collection. The WEI criterion is given by the following theorem.

TheOREm 2.3. [22] A scheme of the form (1.5)-(1.6) satisfying Assumption 2.1 converges for convex conservation laws (1.1) if, for any rarefying pair $\{L, R\}$, there is a constant $\delta>0$ such that the quadrature type inequality

$$
\sum_{j=0}^{n-1}\left(v_{j+1}-v_{j}\right) \bar{g}_{j+1 / 2}+\delta<\int_{L}^{R} f[w ; L, R] d w
$$

holds for all rarefying collections $\left\{v_{j}\right\}_{j=-p}^{n+p}$ with respect to the pair $\{L, R\}$.

Following Osher and Chakravarthy [1, 2], we now introduce the semi-discrete $\alpha$ schemes for $m=2$. Let $g^{E}(\cdot, \cdot)$ be the flux of any $E$-scheme [13], i.e., it is Lipschitz continuous, and, for all $w$ between $w_{j}$ and $w_{j+1}$, it satisfies

$$
\operatorname{sgn}\left(w_{j+1}-w_{j}\right)\left(g^{E}\left(w_{j}, w_{j+1}\right)-f(w)\right) \leq 0 .
$$

Throughout the rest of the paper, we use the following shorthand notations: $f_{j}:=f\left(u_{j}\right), f_{j \pm \frac{1}{2}}:=\Delta f_{j \pm \frac{1}{2}}= \pm\left(f_{j \pm 1}-f_{j}\right), u_{j \pm \frac{1}{2}}:=\Delta u_{j \pm \frac{1}{2}}= \pm\left(u_{j \pm 1}-u_{j}\right)$, and $g_{j+\frac{1}{2}}^{E}:=g^{E}\left(u_{j}, u_{j+1}\right)$.

Denote the flux difference

$$
f_{j+\frac{1}{2}}^{+}:=f_{j+1}-g_{j+\frac{1}{2}}^{E}
$$

and

$$
f_{j+\frac{1}{2}}^{-}:=f_{j}-g_{j+\frac{1}{2}}^{E}
$$

We also set

$$
\left(D f_{j+\frac{1}{2}}\right)^{ \pm}:=f_{j+\frac{1}{2}}^{ \pm} / u_{j+\frac{1}{2}}
$$

Using the notations (2.3)-(2.4), for any $\alpha \in\left(0, \frac{1}{2}\right]$, the numerical flux of an $\alpha$ scheme, when $m=2$, is defined by

$$
g_{j+\frac{1}{2}}=g_{j+\frac{1}{2}}^{E}+\alpha\left(f_{j+\frac{3}{2}}^{-}\right)^{(1)}+\left(\frac{1}{2}-\alpha\right)\left(f_{j+\frac{1}{2}}^{-}\right)^{(0)}+\left(\frac{1}{2}-\alpha\right)\left(f_{j+\frac{1}{2}}^{+}\right)^{(0)}+\alpha\left(f_{j-\frac{1}{2}}^{+}\right)^{(-1)},
$$

where $g^{E}$ is an $E$-flux. The superscripts shown over the $f^{ \pm}$denote flux limited values of $f^{ \pm}$, and are computed as follows: 


$$
\begin{aligned}
& \left(f_{j+\frac{3}{2}}^{-}\right)^{(1)}=\min \bmod \left[f_{j+\frac{3}{2}}^{-}, b f_{j+\frac{1}{2}}^{-}\right], \\
& \left(f_{j+\frac{1}{2}}^{-}\right)^{(0)}=\min \bmod \left[f_{j+\frac{1}{2}}^{-}, b f_{j+\frac{3}{2}}^{-}\right] \\
& \left(f_{j+\frac{1}{2}}^{+}\right)^{(0)}=\min \bmod \left[f_{j+\frac{1}{2}}^{+}, b f_{j-\frac{1}{2}}^{+}\right] \\
& \left(f_{j-\frac{1}{2}}^{+}\right)^{(-1)}=\min \bmod \left[f_{j-\frac{1}{2}}^{+}, b f_{j+\frac{1}{2}}^{+}\right] .
\end{aligned}
$$

In the above, the operator "min mod" is defined by:

$$
\min \bmod (x, y)= \begin{cases}x & \text { if }|x| \leq|y| \text { and } x y>0 \\ y & \text { if }|y|<|x| \text { and } x y>0 \\ 0 & \text { if } x y \leq 0\end{cases}
$$

and $b$ is a "compression" parameter chosen in the range

$$
1<b \leq 1+\frac{1}{2 \alpha}=b_{\max }
$$

The $\alpha$ schemes are defined essentially in terms of one parameter. In the homogeneous case, by various choices of $\alpha$, we can obtain schemes with a wide range of accuracy including a third order accurate TVD scheme. Some special cases are summarized in Table $1[1,2]$. The truncation error (TE) showing in the last column of Table 1 agrees to the corresponding unlimited form. The names of the schemes are based on the names in the literature for the corresponding unlimited ones.

With regard to the orders of the accuracy of $\alpha$ schemes, we make the following remark as discussed in $[1,2]$. The non-TVD or unlimited form of $\alpha$ schemes can be obtained by replacing $\left(f^{ \pm}\right)^{(i)}, i=0, \pm 1$, with their corresponding unlimited $f^{ \pm}$ values in (2.6). Since the parameter $b$ is quite large in all the particular schemes listed in the table, the flux-limited value returned most often will be the unlimited value itself. Thus, for most grid points, the TVD scheme which employs flux limiters will be locally identically to the corresponding unlimited scheme. Therefore, the TE of the unlimited scheme is a good indication of the overall TE of the related TVD scheme.

In the following, for the readability, we provide the numerical fluxes of the three monotone schemes that will be used later on in $(2.6)$ for $g^{E}(\cdot, \cdot)$.

The Lax-Friedrichs flux [12]:

$$
g_{j+\frac{1}{2}}^{L F}=g^{L F}\left(u_{j}, u_{j+1}\right)=\frac{f_{j}+f_{j+1}}{2}-\frac{a}{2} u_{j+\frac{1}{2}}
$$

where $a \geq \max \left|f^{\prime}(w)\right|$. 


\begin{tabular}{cccc}
\hline \hline Value of $\alpha$ & Name of TVD Scheme & $b_{\max }$ & 2nd order TE \\
\hline$\frac{1}{6}$ & Third-Order & 4 & 0 \\
$\frac{1}{2}$ & Fully Upwind & 2 & $-\frac{1}{3} h^{2} \frac{\partial^{3}}{\partial x^{3}} f(u)$ \\
$\frac{1}{4}$ & Fromm's & 3 & $-\frac{1}{12} h^{2} \frac{\partial^{3}}{\partial x^{3}} f(u)$ \\
$\frac{1}{8}$ & Low TE 2nd-order & 5 & $\frac{1}{24} h^{2} \frac{\partial^{3}}{\partial x^{3}} f(u)$ \\
0 & Central & $\infty$ & $\frac{1}{6} h^{2} \frac{\partial^{3}}{\partial x^{3}} f(u)$ \\
$\frac{1}{3}$ & No Name & $\frac{5}{2}$ & $-\frac{1}{6} h^{2} \frac{\partial^{3}}{\partial x^{3}} f(u)$ \\
\hline \hline
\end{tabular}

TABLE 1

Particular Cases of New family of TVD Schemes

The Godunov flux [6]:

$$
\begin{aligned}
g_{j+\frac{1}{2}}^{\text {God }} & =g^{\text {God }}\left(u_{j}, u_{j+1}\right) \\
& =\left\{\begin{array}{lll}
\min _{u_{j} \leq w \leq u_{j+1}} f(w) & \text { when } & u_{j} \leq u_{j+1}, \\
\max _{u_{j} \geq w \geq u_{j+1}} f(w) & \text { when } & u_{j} \geq u_{j+1}
\end{array}\right.
\end{aligned}
$$

The Engquist-Osher flux [5]:

$$
\begin{aligned}
g_{j+\frac{1}{2}}^{E O} & =g^{E O}\left(u_{j}, u_{j+1}\right) \\
& =\int_{0}^{u_{j}} \max \left(f^{\prime}(w), 0\right) d w+\int_{0}^{u_{j+1}} \min \left(f^{\prime}(w), 0\right) d w+f(0) .
\end{aligned}
$$

Let $s$ be a sonic point: $f^{\prime}(s)=0$. Then the Godunov and Engquist-Osher fluxes belong to a subclass of $E$-fluxes that satisfies

$$
g^{E}(x, y)= \begin{cases}f(x) & \text { if } s \leq x \leq y \\ f(y) & \text { if } x \leq y \leq s\end{cases}
$$

The focus of this paper concerns the convergence to the entropy solution of the semi-discrete high resolution $\alpha$ schemes that satisfy the Assumption 2.1, when the building block of the schemes is either the Lax-Friedrichs scheme, or the aforementioned subclass of $E$-schemes. The main result is the following.

THEOREM 2.4. The numerical solutions of the schemes (1.5)-(1.6) ((1.6)(1.7)resp.), for the convex problems (1.1) ((1.2) resp.), converge to the entropy solution provided that the numerical flux $g_{j+\frac{1}{2}}$ is defined by $(2.6), g^{E}(\cdot, \cdot)$ is either the 
numerical flux of the Lax-Friedrichs scheme or that of one of the subclass of E-schemes of which the numerical flux satisfies (2.16).

3. Proof of the main result. The following lemma verifies that the Assumption 2.1 holds for $\alpha$ schemes that we are concerned. 2.1.

Lemma 3.1. The numerical flux $g_{j+\frac{1}{2}}$ defined by (2.6) satisfies the Assumption

Proof. First, let $u_{j}$ be a spatial minimum. Then $f_{j+\frac{1}{2}}^{+} \geq 0, f_{j-\frac{1}{2}}^{+} \leq 0$ and $f_{j+\frac{1}{2}}^{-} \geq 0$ imply that $\left(f_{j+\frac{1}{2}}^{+}\right)^{(0)}=0,\left(f_{j-\frac{1}{2}}^{+}\right)^{(-1)}=0$, as well as

$$
\left(f_{j+\frac{3}{2}}^{-}\right)^{(1)}=\min \bmod \left[f_{j+\frac{3}{2}}^{-}, b f_{j+\frac{1}{2}}^{-}\right] \leq b f_{j+\frac{1}{2}}^{-},
$$

and

$$
\left(f_{j+\frac{1}{2}}^{-}\right)^{(0)}=\min \bmod \left[f_{j+\frac{1}{2}}^{-}, b f_{j+\frac{3}{2}}^{-}\right] \leq f_{j+\frac{1}{2}}^{-} .
$$

With reference to (2.6) and (2.12), we have

$$
\begin{aligned}
g_{j+\frac{1}{2}} & =g_{j+\frac{1}{2}}^{E}+\alpha\left(f_{j+\frac{3}{2}}^{-}\right)^{(1)}+\left(\frac{1}{2}-\alpha\right)\left(f_{j+\frac{1}{2}}^{-}\right)^{(0)} \\
& \leq g_{j+\frac{1}{2}}^{E}+\alpha b f_{j+\frac{1}{2}}^{-}+\left(\frac{1}{2}-\alpha\right) f_{j+\frac{1}{2}}^{-} \\
& \leq g_{j+\frac{1}{2}}^{E}+\left[\alpha\left(1+\frac{1}{2 \alpha}\right)+\frac{1}{2}-\alpha\right] f_{j+\frac{1}{2}}^{-} \\
& =f_{j} .
\end{aligned}
$$

Next, using the fact that $f_{j-\frac{1}{2}}^{-} \leq 0$ and $f_{j+\frac{1}{2}}^{-} \geq 0$, we have $\left(f_{j+\frac{1}{2}}^{-}\right)^{(1)}=0$ and $\left(f_{j-\frac{1}{2}}^{-}\right)^{(0)}=0$. Also $f_{j-\frac{1}{2}}^{+} \leq 0$ implies that

$$
\left(f_{j-\frac{1}{2}}^{+}\right)^{(0)}=\min \bmod \left[f_{j-\frac{1}{2}}^{+}, b f_{j-\frac{3}{2}}^{+}\right] \geq f_{j-\frac{1}{2}}^{+}
$$

and

$$
\left(f_{j-\frac{3}{2}}^{+}\right)^{(-1)}=\min \bmod \left[f_{j-\frac{3}{2}}^{+}, b f_{j-\frac{1}{2}}^{+}\right] \geq b f_{j-\frac{1}{2}}^{+} .
$$

Again, in referencing of (2.6) and (2.12), we have

$$
\begin{aligned}
g_{j-\frac{1}{2}} & =g_{j-\frac{1}{2}}^{E}+\left(\frac{1}{2}-\alpha\right)\left(f_{j-\frac{1}{2}}^{+}\right)^{(0)}+\alpha\left(f_{j-\frac{3}{2}}^{+}\right)^{(-1)} \\
& \geq g_{j-\frac{1}{2}}^{E}+\left(\frac{1}{2}-\alpha\right) f_{j-\frac{1}{2}}^{+}+\alpha b f_{j-\frac{1}{2}}^{+} \\
& \geq f_{j} .
\end{aligned}
$$

In combining of (3.1) and (3.2), we have shown that if $u_{j} \leq u_{j \pm 1}$, then $g_{j+\frac{1}{2}} \leq$ $f_{j} \leq g_{j-\frac{1}{2}}$ as desired.

Second, let $u_{j+1}$ be a spatial maximum. Then we have $f_{j+\frac{3}{2}}^{-} \leq 0$ and $f_{j+\frac{1}{2}}^{-} \geq 0$. Thus $\left(f_{j+\frac{3}{2}}^{-}\right)^{(1)}=0$ and $\left(f_{j+\frac{1}{2}}^{-}\right)^{(0)}=0$. Also the following inequalities 


$$
\left(f_{j+\frac{1}{2}}^{+}\right)^{(0)}=\min \bmod \left[f_{j+\frac{1}{2}}^{+}, b f_{j-\frac{1}{2}}^{+}\right] \leq f_{j+\frac{1}{2}}^{+}
$$

and

$$
\left(f_{j-\frac{1}{2}}^{+}\right)^{(-1)}=\min \bmod \left[f_{j-\frac{1}{2}}^{+}, b f_{j+\frac{1}{2}}^{+}\right] \leq b f_{j+\frac{1}{2}}^{+}
$$

are valid since $f_{j+\frac{1}{2}}^{+} \geq 0$.

Hence,

$$
\begin{aligned}
g_{j+\frac{1}{2}} & =g_{j+\frac{1}{2}}^{E}+\left(\frac{1}{2}-\alpha\right)\left(f_{j+\frac{1}{2}}^{+}\right)^{(0)}+\alpha\left(f_{j-\frac{1}{2}}^{+}\right)^{(-1)} \\
& \leq g_{j+\frac{1}{2}}^{E}+\left(\frac{1}{2}-\alpha\right) f_{j+\frac{1}{2}}^{+}+\alpha b f_{j+\frac{1}{2}}^{+} \\
& \leq f_{j+1} .
\end{aligned}
$$

Replacing $j$ with $j-1$ in the preceding inequality, we see that if $u_{j} \geq u_{j \pm 1}$, then the following

$$
g_{j-\frac{1}{2}} \leq f_{j}
$$

holds.

Also, under the condition of $u_{j}$ is a spatial maximum, it is easy to see that we have $\left(f_{j+\frac{1}{2}}^{+}\right)^{(0)}=0,\left(f_{j-\frac{1}{2}}^{+}\right)^{(-1)}=0, f_{j+\frac{1}{2}}^{-} \leq 0,\left(f_{j+\frac{3}{2}}^{-}\right)^{(1)} \geq b f_{j+\frac{1}{2}}^{-}$, and $\left(f_{j+\frac{1}{2}}^{-}\right)^{(0)} \geq f_{j+\frac{1}{2}}^{-}$. Thus,

$$
\begin{aligned}
g_{j+\frac{1}{2}} & =g_{j+\frac{1}{2}}^{E}+\alpha\left(f_{j+\frac{3}{2}}^{-}\right)^{(1)}+\left(\frac{1}{2}-\alpha\right)\left(f_{j+\frac{1}{2}}^{-}\right)^{(0)} \\
& \geq g_{j+\frac{1}{2}}^{E}+\alpha b f_{j+\frac{1}{2}}^{-}+\left(\frac{1}{2}-\alpha\right) f_{j+\frac{1}{2}}^{-} \\
& \geq f_{j} .
\end{aligned}
$$

Inequalities (3.1), (3.2), (3.3) and (3.4) verify that $\alpha$ schemes indeed satisfy the Assumption 2.1. Thus, we have completed the proof of the lemma.

Now, we define

$$
\bar{f}[c, d]:=\int_{c}^{d}\{f[w ; c, d]-f(w)\} d w
$$

for $c<d$, and

$$
S_{j+\frac{1}{2}}:=\int_{v_{j}}^{v_{j+1}}\left[\bar{g}_{j+\frac{1}{2}}-f(w)\right] d w .
$$

In the following we present a very important lemma, which underlines the entropy property of the schemes.

Lemma 3.2. The numerical solutions of the schemes (1.5)-(1.6) ((1.6)(1.7)resp.), for the convex problems (1.1) ((1.2) resp.), satisfy

$$
\sum_{j=0}^{n-1}\left(S_{j+\frac{1}{2}}-\bar{f}\left[v_{j}, v_{j+1}\right]\right) \leq 0,
$$


provided that the numerical flux $g_{j+\frac{1}{2}}$ is defined by $(2.6), g^{E}(\cdot, \cdot)$ is either the numerical flux of the Lax-Friedrichs scheme or that of one of the subclass of E-schemes of which the numerical flux satisfies (2.16).

Proof. Let $\{L, R\}$ be an arbitrary rarefying pair $\left\{v_{j}\right\}_{j=-p}^{n+p}$ and $\bar{g}_{j+\frac{1}{2}}$ be as in Theorem 2.3. We keep the same notations $f_{j+\frac{1}{2}}^{ \pm}$, and $\left(D f_{j+\frac{1}{2}}\right)^{ \pm}$for $\left\{v_{j}\right\}$ instead of $\left\{u_{j}\right\}$ here. We also use the following notation for the divided difference:

$$
f_{j+\frac{1}{2}}^{\prime}:=\frac{f_{j+1}-f_{j}}{v_{j+1}-v_{j}}
$$

To justify the inequality (3.7), note that the numerical flux (2.6), evaluated at a rarefying collection $\left\{v_{j}\right\}_{j=-p}^{n+p}$ with respect to any rarefying pair $\{L, R\}$, can be written as:

$$
\bar{g}_{j+\frac{1}{2}}=g_{j+\frac{1}{2}}^{E}+\alpha\left(f_{j+\frac{3}{2}}^{-}\right)^{(1)}+\left(\frac{1}{2}-\alpha\right)\left(f_{j+\frac{1}{2}}^{-}\right)^{(0)}+\left(\frac{1}{2}-\alpha\right)\left(f_{j+\frac{1}{2}}^{+}\right)^{(0)}+\alpha\left(f_{j-\frac{1}{2}}^{+}\right)^{(-1)} .
$$

Now, by (2.3) and (2.4), we have

$$
\int_{v_{j}}^{v_{j+1}}\left[g_{j+\frac{1}{2}}^{E}-\frac{f_{j+1}+f_{j}}{2}\right] d w=-\frac{f_{j+\frac{1}{2}}^{-}+f_{j+\frac{1}{2}}^{+}}{2} v_{j+\frac{1}{2}} .
$$

Using the fact that

$$
\begin{gathered}
\left(f_{-\frac{1}{2}}^{+}\right)^{(-1)}=0, \quad\left(f_{\frac{1}{2}}^{+}\right)^{(0)}=0 \leq f_{\frac{1}{2}}^{+}, \quad\left(f_{\frac{3}{2}}^{-}\right)^{(1)} \leq f_{\frac{3}{2}}^{-}, \quad\left(f_{\frac{1}{2}}^{-}\right)^{(0)} \leq f_{\frac{1}{2}}^{-} ; \\
\left(f_{n+\frac{1}{2}}^{-}\right)^{(1)}=0, \quad\left(f_{n-\frac{1}{2}}^{-}\right)^{(0)}=0 \leq f_{n-\frac{1}{2}}^{-} ; \\
\left(f_{n-\frac{1}{2}}^{+}\right)^{(0)} \leq f_{n-\frac{1}{2}}^{+}, \quad\left(f_{n-\frac{3}{2}}^{+}\right)^{(-1)} \leq f_{n-\frac{3}{2}}^{+} ;
\end{gathered}
$$

and for all $j, 1 \leq j \leq n-2$,

$$
\begin{aligned}
& \left(f_{j+\frac{3}{2}}^{-}\right)^{(1)} \leq f_{j+\frac{3}{2}}^{-}, \quad\left(f_{j+\frac{1}{2}}^{-}\right)^{(0)} \leq f_{j+\frac{1}{2}}^{-} ; \\
& \left(f_{j+\frac{1}{2}}^{+}\right)^{(0)} \leq f_{j+\frac{1}{2}}^{+}, \quad\left(f_{j-\frac{1}{2}}^{+}\right)^{(-1)} \leq f_{j-\frac{1}{2}}^{+} ;
\end{aligned}
$$

we obtain the following estimation. 


$$
\begin{aligned}
& \sum_{j=0}^{n-1}\left(S_{j+\frac{1}{2}}-\bar{f}\left[v_{j}, v_{j+1}\right]\right)=\sum_{j=0}^{n-1}\left(\bar{g}_{j+\frac{1}{2}}-\frac{f_{j}+f_{j+1}}{2}\right) v_{j+\frac{1}{2}} \\
= & \sum_{j=0}^{n-1}\left\{-\frac{1}{2}\left[f_{j+\frac{1}{2}}^{-}+f_{j+\frac{1}{2}}^{+}\right] v_{j+\frac{1}{2}}\right. \\
+ & {\left.\left[\alpha\left(f_{j+\frac{3}{2}}^{-}\right)^{(1)}+\left(\frac{1}{2}-\alpha\right)\left(f_{j+\frac{1}{2}}^{-}\right)^{(0)}+\left(\frac{1}{2}-\alpha\right)\left(f_{j+\frac{1}{2}}^{+}\right)^{(0)}+\alpha\left(f_{j-\frac{1}{2}}^{+}\right)^{(-1)}\right] v_{j+\frac{1}{2}}\right\} }
\end{aligned}
$$$$
\leq \sum_{j=1}^{n-2}\left\{-\frac{1}{2}\left[f_{j+\frac{1}{2}}^{-}+f_{j+\frac{1}{2}}^{+}\right] v_{j+\frac{1}{2}}\right.
$$$$
\left.+\left[\alpha f_{j+\frac{3}{2}}^{-}+\left(\frac{1}{2}-\alpha\right) f_{j+\frac{1}{2}}^{-}+\left(\frac{1}{2}-\alpha\right) f_{j+\frac{1}{2}}^{+}+\alpha f_{j-\frac{1}{2}}^{+}\right] v_{j+\frac{1}{2}}\right\}
$$$$
-\frac{1}{2}\left[f_{\frac{1}{2}}^{-}+f_{\frac{1}{2}}^{+}\right] v_{\frac{1}{2}}+\left[\alpha f_{\frac{3}{2}}^{-}+\left(\frac{1}{2}-\alpha\right) f_{\frac{1}{2}}^{-}+\left(\frac{1}{2}-\alpha\right) f_{\frac{1}{2}}^{+}\right] v_{\frac{1}{2}}
$$$$
-\frac{1}{2}\left[f_{n-\frac{1}{2}}^{-}+f_{n-\frac{1}{2}}^{+}\right] v_{n-\frac{1}{2}}+\left[\left(\frac{1}{2}-\alpha\right) f_{n-\frac{1}{2}}^{-}+\left(\frac{1}{2}-\alpha\right) f_{n-\frac{1}{2}}^{+}+\alpha f_{n-\frac{3}{2}}^{+}\right] v_{n-\frac{1}{2}}
$$$$
\leq \alpha\left\{\sum_{j=1}^{n-2}\left[f_{j-\frac{1}{2}}^{+}-f_{j+\frac{1}{2}}^{+}+f_{j+\frac{3}{2}}^{-}-f_{j+\frac{1}{2}}^{-}\right] v_{j+\frac{1}{2}}\right.
$$$$
\left.+\left[-f_{\frac{1}{2}}^{+}+f_{\frac{3}{2}}^{-}-f_{\frac{1}{2}}^{-}\right] v_{\frac{1}{2}}+\left[-f_{n-\frac{1}{2}}^{-}-f_{n-\frac{1}{2}}^{+}+f_{n-\frac{3}{2}}^{+}\right] v_{n-\frac{1}{2}}\right\}
$$$$
:=\alpha\{A+B\} \text {, }
$$

with

$$
\begin{aligned}
A & =\sum_{j=1}^{n-2}\left[f_{j-\frac{1}{2}}^{+}-f_{j+\frac{1}{2}}^{+}+f_{j+\frac{3}{2}}^{-}-f_{j+\frac{1}{2}}^{-}\right] v_{j+\frac{1}{2}} \\
& =\sum_{j=1}^{n-2}-\left[f_{j+\frac{1}{2}}^{+}+f_{j+\frac{1}{2}}^{-}\right] v_{j+\frac{1}{2}}+\sum_{j=1}^{n-2}\left[f_{j-\frac{1}{2}}^{+}+f_{j+\frac{3}{2}}^{-}\right] v_{j+\frac{1}{2}}
\end{aligned}
$$

and

$$
B=\left[-f_{\frac{1}{2}}^{+}+f_{\frac{3}{2}}^{-}-f_{\frac{1}{2}}^{-}\right] v_{\frac{1}{2}}+\left[-f_{n-\frac{1}{2}}^{-}-f_{n-\frac{1}{2}}^{+}+f_{n-\frac{3}{2}}^{+}\right] v_{n-\frac{1}{2}} .
$$

First, in the case of Lax-Friedrichs flux, noticing that (2.3)-(2.5), (2.13) and (3.8) imply the equality

$$
\left(D f_{j+\frac{1}{2}}\right)^{ \pm}=\left(a \pm f_{j+\frac{1}{2}}^{\prime}\right) / 2,
$$


we then have

$$
\begin{aligned}
A & =\sum_{j=1}^{n-2}-\left[\left(D f_{j+\frac{1}{2}}\right)^{+}+\left(D f_{j+\frac{1}{2}}\right)^{-}\right] v_{j+\frac{1}{2}}^{2} \\
& +\sum_{j=1}^{n-2}\left[\left(D f_{j-\frac{1}{2}}\right)^{+} v_{j-\frac{1}{2}} v_{j+\frac{1}{2}}+\left(D f_{j+\frac{3}{2}}\right)^{-} v_{j+\frac{1}{2}} v_{j+\frac{3}{2}}\right] \\
& =\sum_{j=1}^{n-2}\left[-a v_{j+\frac{1}{2}}^{2}+a v_{j-\frac{1}{2}} v_{j+\frac{1}{2}}\right]+\frac{1}{2} \sum_{j=1}^{n-2}\left[f_{j-\frac{1}{2}}^{\prime}-f_{j+\frac{1}{2}}^{\prime}\right] v_{j-\frac{1}{2}} v_{j+\frac{1}{2}} \\
& -\frac{1}{2}\left(a-f_{\frac{3}{2}}^{\prime}\right) v_{\frac{1}{2}} v_{\frac{3}{2}}+\frac{1}{2}\left(a-f_{n-\frac{1}{2}}^{\prime}\right) v_{n-\frac{3}{2}} v_{n-\frac{1}{2}} \\
& \leq \frac{a}{2} v_{\frac{1}{2}}^{2}-\frac{a}{2} v_{n-\frac{3}{2}}^{2}-\frac{1}{2}\left(a-f_{\frac{3}{2}}^{\prime}\right) v_{\frac{1}{2}} v_{\frac{3}{2}}+\frac{1}{2}\left(a-f_{n-\frac{1}{2}}^{\prime}\right) v_{n-\frac{3}{2}} v_{n-\frac{1}{2}}
\end{aligned}
$$

and

$$
\begin{aligned}
B & =-\left[\left(D f_{\frac{1}{2}}\right)^{+}+\left(D f_{\frac{1}{2}}\right)^{-}\right] v_{\frac{1}{2}}^{2}+\left(D f_{\frac{3}{2}}\right)^{-} v_{\frac{1}{2}} v_{\frac{3}{2}} \\
& -\left[\left(D f_{n-\frac{1}{2}}\right)^{+}+\left(D f_{n-\frac{1}{2}}\right)^{-}\right] v_{n-\frac{1}{2}}^{2}+\left(D f_{n-\frac{3}{2}}\right)^{+} v_{n-\frac{3}{2}} v_{n-\frac{1}{2}} \\
& =-a v_{\frac{1}{2}}^{2}+\frac{1}{2}\left(a-f_{\frac{3}{2}}^{\prime}\right) v_{\frac{1}{2}} v_{\frac{3}{2}}-a v_{n-\frac{1}{2}}^{2}+\frac{1}{2}\left(a+f_{n-\frac{3}{2}}^{\prime}\right) v_{n-\frac{3}{2}} v_{n-\frac{1}{2}} .
\end{aligned}
$$

Combining (3.15) and (3.16), we have the following inequality

$$
\begin{aligned}
A+B & \leq-\frac{a}{2} v_{\frac{1}{2}}^{2}-\frac{a}{2} v_{n-\frac{3}{2}}^{2}+a v_{n-\frac{3}{2}} v_{n-\frac{1}{2}} \\
& -\frac{1}{2} f_{n-\frac{1}{2}}^{\prime} v_{n-\frac{3}{2}} v_{n-\frac{1}{2}}-a v_{n-\frac{1}{2}}^{2}+\frac{1}{2} f_{n-\frac{3}{2}}^{\prime} v_{n-\frac{3}{2}} v_{n-\frac{1}{2}} \\
& \leq-\frac{a}{2} v_{\frac{1}{2}}^{2}-\frac{a}{2} v_{n-\frac{1}{2}}^{2}-\frac{1}{2}\left(f_{n-\frac{1}{2}}^{\prime}-f_{n-\frac{3}{2}}^{\prime}\right) v_{n-\frac{3}{2}} v_{n-\frac{1}{2}} \\
& \leq 0
\end{aligned}
$$

as desired.

Next, we deal with the case that $g^{E}(\cdot, \cdot)$ is the numerical flux of an $E$-scheme satisfying (2.16). Without loss of generality, let $s$ be the sonic point such that $v_{k} \leq s \leq$ $v_{k+1}$ for some integer $k$ with $0 \leq k \leq n-1$. We denote $f_{s}:=f\left(v_{s}\right), v_{s+\frac{1}{2}}:=v_{k+1}-v_{s}$, $v_{s-\frac{1}{2}}:=v_{s}-v_{k}, f_{s+\frac{1}{2}}^{\prime}:=\left(f_{k+1}-f_{s}\right) / v_{s+\frac{1}{2}}, f_{s-\frac{1}{2}}^{\prime}:=\left(f_{s}-f_{k}\right) / v_{s-\frac{1}{2}}$. Then,

$$
\begin{gathered}
f_{j+\frac{1}{2}}^{+}=0 \quad \text { for } \quad 0 \leq j \leq k-1 \\
f_{j+\frac{1}{2}}^{+}=f_{j+\frac{1}{2}}^{\prime} v_{j+\frac{1}{2}} \quad \text { for } \quad n-1 \geq j \geq k+1 \\
f_{j+\frac{1}{2}}^{-}=0 \quad \text { for } \quad n-1 \geq j \geq k+1
\end{gathered}
$$


and

$$
f_{j+\frac{1}{2}}^{-}=-f_{j+\frac{1}{2}}^{\prime} v_{j+\frac{1}{2}} \quad \text { for } \quad 0 \leq j \leq k-1 .
$$

Again, by (3.9) and (3.10), we have

$$
\begin{aligned}
& \sum_{j=0}^{n-1}\left(S_{j+\frac{1}{2}}-\bar{f}\left[v_{j}, v_{j+1}\right]\right)=\sum_{j=0}^{n-1}\left(\bar{g}_{j+\frac{1}{2}}-\frac{f_{j}+f_{j+1}}{2}\right) v_{j+\frac{1}{2}} \\
= & \sum_{j=0}^{n-1}\left\{-\frac{1}{2}\left[f_{j+\frac{1}{2}}^{-}+f_{j+\frac{1}{2}}^{+}\right] v_{j+\frac{1}{2}}\right. \\
+ & {\left.\left[\alpha\left(f_{j+\frac{3}{2}}^{-}\right)^{(1)}+\left(\frac{1}{2}-\alpha\right)\left(f_{j+\frac{1}{2}}^{-}\right)^{(0)}+\left(\frac{1}{2}-\alpha\right)\left(f_{j+\frac{1}{2}}^{+}\right)^{(0)}+\alpha\left(f_{j-\frac{1}{2}}^{+}\right)^{(-1)}\right] v_{j+\frac{1}{2}}\right\} . }
\end{aligned}
$$

To derive the desired inequality, we have split the above estimation into three parts: $P_{1}, P_{2}$ and $P_{3}$ as presented in the follows.

$$
\begin{aligned}
& \sum_{j=0}^{n-1}\left(S_{j+\frac{1}{2}}-\bar{f}\left[v_{j}, v_{j+1}\right]\right) \\
= & \sum_{j=0}^{k-2}\left(S_{j+\frac{1}{2}}-\bar{f}\left[v_{j}, v_{j+1}\right]\right)+\sum_{j=k+2}^{n-1}\left(S_{j+\frac{1}{2}}-\bar{f}\left[v_{j}, v_{j+1}\right]\right) \\
+ & \sum_{j=k-1}^{k+1}\left(S_{j+\frac{1}{2}}-\bar{f}\left[v_{j}, v_{j+1}\right]\right) \\
:= & P_{1}+P_{2}+P_{3},
\end{aligned}
$$

where

$$
\begin{aligned}
P_{1} & =\sum_{j=0}^{k-2}\left(S_{j+\frac{1}{2}}-\bar{f}\left[v_{j}, v_{j+1}\right]\right) \\
& =\sum_{j=0}^{k-2}\left\{-\frac{1}{2} f_{j+\frac{1}{2}}^{-} v_{j+\frac{1}{2}}+\left[\alpha\left(f_{j+\frac{3}{2}}^{-}\right)^{(1)}+\left(\frac{1}{2}-\alpha\right)\left(f_{j+\frac{1}{2}}^{-}\right)^{(0)}\right] v_{j+\frac{1}{2}}\right\} \\
& \leq \alpha \sum_{j=0}^{k-2}\left[f_{j+\frac{3}{2}}^{-}-f_{j+\frac{1}{2}}^{-}\right] v_{j+\frac{1}{2}} \\
& =\alpha \sum_{j=0}^{k-2}\left[-f_{j+\frac{3}{2}}^{\prime} v_{j+\frac{3}{2}} v_{j+\frac{1}{2}}+f_{j+\frac{1}{2}}^{\prime} v_{j+\frac{1}{2}}^{2}\right] \\
& \leq \sum_{j=0}^{k-2} \frac{1}{2} \alpha f_{j+\frac{3}{2}}^{\prime}\left(v_{j+\frac{3}{2}}-v_{j+\frac{1}{2}}\right)^{2}+\frac{1}{2} \alpha f_{\frac{1}{2}}^{\prime} v_{\frac{1}{2}}^{2}-\frac{1}{2} \alpha f_{k-\frac{1}{2}}^{\prime} v_{k-\frac{1}{2}}^{2} \\
& \leq-\frac{1}{2} \alpha f_{k-\frac{1}{2}}^{\prime} v_{k-\frac{1}{2}}^{2}
\end{aligned}
$$




$$
\begin{aligned}
P_{2} & =\sum_{j=k+2}^{n-1}\left(S_{j+\frac{1}{2}}-\bar{f}\left[v_{j}, v_{j+1}\right]\right) \\
& =\sum_{j=k+2}^{n-1}\left\{-\frac{1}{2} f_{j+\frac{1}{2}}^{+} v_{j+\frac{1}{2}}+\left[\left(\frac{1}{2}-\alpha\right)\left(f_{j+\frac{1}{2}}^{+}\right)^{(0)}+\alpha\left(f_{j-\frac{1}{2}}^{+}\right)^{(-1)}\right] v_{j+\frac{1}{2}}\right\} \\
& \leq \sum_{j=k+2}^{n-1} \alpha\left[f_{j-\frac{1}{2}}^{+} v_{j+\frac{1}{2}}-f_{j+\frac{1}{2}}^{+} v_{j+\frac{1}{2}}\right] \\
& =\sum_{j=k+2}^{n-1} \alpha\left[f_{j-\frac{1}{2}}^{\prime} v_{j-\frac{1}{2}}-f_{j+\frac{1}{2}}^{\prime} v_{j+\frac{1}{2}}\right] v_{j+\frac{1}{2}} \\
& \leq \sum_{j=k+2}^{n-1}-\frac{1}{2} \alpha f_{j-\frac{1}{2}}^{\prime}\left(v_{j-\frac{1}{2}}-v_{j+\frac{1}{2}}\right)^{2}+\frac{1}{2} \alpha f_{k+\frac{3}{2}}^{\prime} v_{k+\frac{3}{2}}^{2}-\frac{1}{2} \alpha f_{n-\frac{1}{2}}^{\prime} v_{n-\frac{1}{2}}^{2} \\
& \leq \frac{1}{2} \alpha f_{k+\frac{3}{2}}^{\prime} v_{k+\frac{3}{2}}^{2},
\end{aligned}
$$

and $P_{3}$ is the sum of three terms when $j=k-1, k, k+1$, i.e.,

$$
\begin{aligned}
P_{3} & =\sum_{j=k-1}^{k+1}\left(S_{j+\frac{1}{2}}-\bar{f}\left[v_{j}, v_{j+1}\right]\right) \\
& =\sum_{j=k-1}^{k+1}\left\{-\frac{1}{2}\left[f_{j+\frac{1}{2}}^{-}+f_{j+\frac{1}{2}}^{+}\right] v_{j+\frac{1}{2}}\right. \\
& \left.+\left[\alpha\left(f_{j+\frac{3}{2}}^{-}\right)^{(1)}+\left(\frac{1}{2}-\alpha\right)\left(f_{j+\frac{1}{2}}^{-}\right)^{(0)}+\left(\frac{1}{2}-\alpha\right)\left(f_{j+\frac{1}{2}}^{+}\right)^{(0)}+\alpha\left(f_{j-\frac{1}{2}}^{+}\right)^{(-1)}\right] v_{j+\frac{1}{2}}\right\} \\
& \leq \alpha\left\{\left[f_{k+\frac{1}{2}}^{-}-f_{k-\frac{1}{2}}^{-}\right] v_{k-\frac{1}{2}}+\left[-f_{k+\frac{1}{2}}^{+}-f_{k+\frac{1}{2}}^{-}\right] v_{k+\frac{1}{2}}+\left[f_{k+\frac{1}{2}}^{+}-f_{k+\frac{3}{2}}^{+}\right] v_{k+\frac{3}{2}}\right\} \\
& =\alpha\left\{\left[-f_{s-\frac{1}{2}}^{\prime} v_{s-\frac{1}{2}}+f_{k-\frac{1}{2}}^{\prime} v_{k-\frac{1}{2}}\right] v_{k-\frac{1}{2}}+\left[-f_{s+\frac{1}{2}}^{\prime} v_{s+\frac{1}{2}}+f_{s-\frac{1}{2}}^{\prime} v_{s-\frac{1}{2}}\right] v_{k+\frac{1}{2}}\right. \\
& \left.+\left[f_{s+\frac{1}{2}}^{\prime} v_{s+\frac{1}{2}}-f_{k+\frac{3}{2}}^{\prime} v_{k+\frac{3}{2}}\right] v_{k+\frac{3}{2}}\right\} \\
& =\alpha\left\{-f_{s-\frac{1}{2}}^{\prime} v_{s-\frac{1}{2}} v_{k-\frac{1}{2}}+f_{k-\frac{1}{2}}^{\prime} v_{k-\frac{1}{2}}^{2}-f_{s+\frac{1}{2}}^{\prime} v_{s+\frac{1}{2}} v_{k+\frac{1}{2}}\right. \\
& \left.+f_{s-\frac{1}{2}}^{\prime} v_{s-\frac{1}{2}} v_{s+\frac{1}{2}}+f_{s-\frac{1}{2}}^{\prime} v_{s-\frac{1}{2}}^{2}+f_{s+\frac{1}{2}}^{\prime} v_{s+\frac{1}{2}} v_{k+\frac{3}{2}}-f_{k+\frac{3}{2}}^{\prime} v_{k+\frac{3}{2}}^{2}\right\},
\end{aligned}
$$

where we have used that fact that $v_{k+\frac{1}{2}}=v_{s-\frac{1}{2}}+v_{s+\frac{1}{2}}$.

Then, we group some of the terms of $P_{3}$ into two part: $P_{3_{1}}$ and $P_{3_{2}}$ with the following estimations. 


$$
\begin{aligned}
& \frac{1}{\alpha} P_{3_{1}}=-\frac{1}{2} f_{s+\frac{1}{2}}^{\prime} v_{s+\frac{1}{2}} v_{k+\frac{1}{2}}+f_{s+\frac{1}{2}}^{\prime} v_{s+\frac{1}{2}} v_{k+\frac{3}{2}}-\frac{1}{2} f_{k+\frac{3}{2}}^{\prime} v_{k+\frac{3}{2}}^{2} \\
= & -\frac{1}{2} f_{s+\frac{1}{2}}^{\prime} v_{s+\frac{1}{2}} v_{k+\frac{1}{2}}+f_{s+\frac{1}{2}}^{\prime} v_{s+\frac{1}{2}} v_{k+\frac{3}{2}}-\frac{1}{2} f_{k+\frac{3}{2}}^{\prime} v_{k+\frac{3}{2}}^{2}-\frac{1}{2} f_{s+\frac{1}{2}}^{\prime} v_{s+\frac{1}{2}}^{2}+\frac{1}{2} f_{s+\frac{1}{2}}^{\prime} v_{s+\frac{1}{2}}^{2} \\
\leq & -\frac{1}{2} f_{s+\frac{1}{2}}^{\prime} v_{s+\frac{1}{2}}\left(v_{k+\frac{1}{2}}-v_{s+\frac{1}{2}}\right)-\frac{1}{2} f_{s+\frac{1}{2}}^{\prime}\left(v_{k+\frac{3}{2}}-v_{s+\frac{1}{2}}\right)^{2} \\
= & -\frac{1}{2} f_{s+\frac{1}{2}}^{\prime} v_{s+\frac{1}{2}} v_{s-\frac{1}{2}}-\frac{1}{2} f_{s+\frac{1}{2}}^{\prime}\left(v_{k+\frac{3}{2}}-v_{s+\frac{1}{2}}\right)^{2} \\
\leq & 0
\end{aligned}
$$

and

$$
\begin{aligned}
\frac{1}{\alpha} P_{3_{2}} & =-f_{s-\frac{1}{2}}^{\prime} v_{s-\frac{1}{2}} v_{k-\frac{1}{2}}-\frac{1}{2} f_{s+\frac{1}{2}}^{\prime} v_{s+\frac{1}{2}} v_{k+\frac{1}{2}}+\frac{1}{2} f_{k-\frac{1}{2}}^{\prime} v_{k-\frac{1}{2}}^{2} \\
& +f_{s-\frac{1}{2}}^{\prime} v_{s-\frac{1}{2}} v_{s+\frac{1}{2}}+f_{s-\frac{1}{2}}^{\prime} v_{s-\frac{1}{2}}^{2} \\
& \leq-\frac{1}{2} f_{s+\frac{1}{2}}^{\prime} v_{s+\frac{1}{2}} v_{k+\frac{1}{2}}+\frac{1}{2} f_{s-\frac{1}{2}}^{\prime} v_{s-\frac{1}{2}}^{2} \\
& \leq 0
\end{aligned}
$$

where we have used the relation

$$
\begin{aligned}
& \frac{1}{2} f_{k-\frac{1}{2}}^{\prime} v_{k-\frac{1}{2}}^{2}+\frac{1}{2} f_{s-\frac{1}{2}}^{\prime} v_{s-\frac{1}{2}}^{2}-f_{s-\frac{1}{2}}^{\prime} v_{s-\frac{1}{2}} v_{k-\frac{1}{2}} \\
\leq & \frac{1}{2} f_{s-\frac{1}{2}}^{\prime}\left(v_{s-\frac{1}{2}}-v_{k-\frac{1}{2}}\right)^{2} \\
\leq & 0 .
\end{aligned}
$$

Now, combining (3.18)-(3.24) we have arrived the desired inequality

$$
\begin{aligned}
& \sum_{j=0}^{n-1}\left(S_{j+\frac{1}{2}}-\bar{f}\left[v_{j}, v_{j+1}\right]\right) \\
= & P_{1}+P_{2}+P_{3} \\
\leq & 0
\end{aligned}
$$

and the lemma has been proved.

Finally, we give the proof of the main theorem. The argument is essentially the same as the one given in [7]. We present here for completeness.

Proof of Theorem 2.4. With the lemmas 3.1 and 3.2, and by the Theorem 2.3, to prove the convergence of the schemes (1.5) with the flux $g$ defined by (1.6), it suffices to show that for any rarefying pair $\{L, R\}$, there exists a $\delta>0$ such that for any rarefying collection $\left\{v_{j}\right\}_{j=-p}^{n+p}$ with respect to the pair, the inequality (2.1):

$$
\sum_{j=0}^{n-1}\left(v_{j+1}-v_{j}\right) \bar{g}_{j+1 / 2}+\delta<\int_{L}^{R} f[w ; L, R] d w
$$


holds. Using (3.5) and (3.6), we rewrite the above inequality as

$$
\sum_{j=0}^{n-1} S_{j+\frac{1}{2}}<\bar{f}[L, R]-\delta
$$

Now, by the convexity of $f$, the following inequality holds

$$
\bar{f}[x, y]+\bar{f}[y, z] \leq \bar{f}[x, z] \text { for } \quad x \leq y \leq z .
$$

This and (3.7) yield

$$
\sum_{j=0}^{n-1} S_{j+\frac{1}{2}} \leq \sum_{j=0}^{n-1} \bar{f}\left[v_{j}, v_{j+1}\right] \leq \bar{f}\left[L, v_{i}\right]+\bar{f}\left[v_{i}, R\right] \leq \bar{f}[L, R]
$$

Assume that for each $\delta>0$ there exists a rarefying collection $\left\{v_{j}\right\}_{j=-p}^{n+p}$, with respect to the pair $\{L, R\}$ for some positive integer $n$, violates (3.26). Then (3.28) implies that there exists a sequence of rarefying collections

$\left\{v_{j}^{\nu}, j=-p,-p+1, \cdots, n^{\nu}+p\right\}_{\nu=1}^{\infty}$ with respect to the pair $\{L, R\}$ such that

$$
\lim _{\nu \rightarrow \infty} \sum_{j=0}^{n^{\nu}-1} S_{j+\frac{1}{2}}^{\nu}=\lim _{\nu \rightarrow \infty} \sum_{j=0}^{n^{\nu}-1} \bar{f}\left[v_{j}^{\nu}, v_{j+1}^{\nu}\right]=\bar{f}[L, R]
$$

This implies that there exists a sequence of integers $\left\{j^{\nu}\right\}$ satisfying $0 \leq j^{\nu} \leq n^{\nu}-1$ such that

$$
\lim _{\nu \rightarrow \infty} v_{j^{\nu}}^{\nu}=L \quad \text { and } \quad \lim _{\nu \rightarrow \infty} v_{j^{\nu}+1}^{\nu}=R
$$

For, otherwise, there would exist a constant $\rho>0$, a subsequence of the rarefying collections, still denoted by $\left\{v_{j}^{\nu}, j=-p,-p+1, \cdots, n^{\nu}+p\right\}_{\nu=1}^{\infty}$, and a sequence of integers $\left\{i^{\nu}\right\}$ satisfying $1 \leq i^{\nu} \leq n^{\nu}-1$ such that

$L+\rho<v_{i^{\nu}}^{\nu}<R-\rho$. Then the convexity of $f$, the definition of rarefying pairs, and the inequality (3.28) would imply

$$
\begin{aligned}
& \sum_{j=0}^{n^{\nu}-1} \bar{f}\left[v_{j}^{\nu}, v_{j+1}^{\nu}\right] \\
\leq & \bar{f}\left[L, v_{i^{\nu}}^{\nu}\right]+\bar{f}\left[v_{i^{\nu}}^{\nu}, R\right] \\
\leq & H_{\rho}:=\max (\bar{f}[L, L+\rho]+\bar{f}[L+\rho, R], \bar{f}[L, R-\rho]+\bar{f}[R-\rho, R]) \\
< & \bar{f}[L, R] .
\end{aligned}
$$

This would contradict (3.29) because $H_{\rho}$ is independent of $\nu$. Hence, we have confirmed the existence of a sequence $\left\{j^{\nu}\right\}$ satisfying (3.30). Now, combining (3.27)(3.30), we obtain 


$$
\begin{aligned}
\bar{f}[L, R] & =\lim _{\nu \rightarrow \infty} \sum_{j=0}^{n^{\nu}-1} S_{j+\frac{1}{2}}^{\nu} \\
& =\lim _{\nu \rightarrow \infty}\left(\sum_{j=0}^{j^{\nu}-1} S_{j+\frac{1}{2}}^{\nu}+S_{j^{\nu}+\frac{1}{2}}^{\nu}+\sum_{j=j^{\nu}+1}^{n^{\nu}-1} S_{j+\frac{1}{2}}^{\nu}\right) \\
& \leq \lim _{\nu \rightarrow \infty}\left(\bar{f}\left[L, v_{j^{\nu}}^{\nu}\right]+S_{j^{\nu}+\frac{1}{2}}^{\nu}+\bar{f}\left[v_{j^{\nu}+1}^{\nu}, R\right]\right) \\
& =\lim _{\nu \rightarrow \infty} S_{j^{\nu}+\frac{1}{2}}^{\nu} \\
& \leq \bar{f}[L, R] .
\end{aligned}
$$

This implies

$$
\lim _{\nu \rightarrow \infty} S_{j^{\nu}+\frac{1}{2}}^{\nu}=\bar{f}[L, R]
$$

However, applying (3.30) and the definition of the rarefying collections with respect to the rarefying pair $\{L, R\}$ to $(2.6)$, we get

$$
\lim _{\nu \rightarrow \infty} \bar{g}_{j^{\nu}+\frac{1}{2}}^{\nu}=g^{E}(L, R)
$$

and hence, by (3.6),

$$
\lim _{\nu \rightarrow \infty} S_{j^{\nu}+\frac{1}{2}}^{\nu} \leq 0
$$

This contradicts (3.33) since $\bar{f}[L, R]>0$ by the definition of the rarefying pair $\{L, R\}$. Thus, we have finished the proof.

Acknowledgments. The author wishes to thank the referees for their careful reading of the manuscript and helpful comments, which have been incorporated into revision of this manuscript.

\section{REFERENCES}

[1] S. Osher and S. Chakravarthy, Very High Order Accurate TVD Schemes, Journal of Oscillation theory, computation, and methods of compensated compactness, (1986), pp. 229-274.

[2] S. Chakravarthy and S. Osher, A New Class of High Accuracy TVD Schemes for Hyperbolic Conservation Laws, AIAA paper, 1-11, 23rd Aerospace Science Meeting (1985), Reno, Nevada.

[3] S. Chakravarthy And S. Osher, High resolution applications of the Osher upwind scheme for the Euler equations, AIAA paper presented at 6th CFD conference 1983.

[4] R. J. DiPernA, Convergence of approximate solutions to conservation laws, Arch. Rational Mech. Aual., 82 (1983), pp. 27-70.

[5] B. Engquist AND S. Osher, Stable and entropy satisfying approximations for transonic flow calculations, Math. Comp., 34 (1980), pp. 45-75.

[6] S. K. Godunov, Finite-difference method for numerical computation of discontinuous solutions of the equations of fluid dynamics, Mat. Sbornik, 47 (1959), pp. 271-306.

[7] N. JiAng AND H. YAng, On convergence of Semi-discrete High Resolution Schemes with van Leer's flux limiter for Conservation laws, Methods and Applications of Analysis, 12:1 (2005), pp. 89-102.

[8] N. JiAng, On the Convergence of Fully-discrete High-Resolution Schemes with van Leer's flux limiter for Conservation laws, Methods and Applications of Analysis, 16:3 (2009), pp. 403422 . 
[9] N. JIANG, On the convergence of Semi-discrete High Resolution Schemes with Superbee flux limiter for Conservation laws, Series in Contemporary Applied Mathematics CAM 18, Hyperbolic Problems (Theory, Numerics and Applications), 2 (2012), pp. 431-438.

[10] S. N. KRUZKov, first order quasilinear equations in several independent variables, Math. USSR Sb., 47 (1970), pp. 217-243.

[11] P. Lax And B. Wendroff, Systems of conservation laws, Comm. Pure Appl. Math., 13 (1960), pp. $217-237$.

[12] P. LAx, Hyperbolic Systems of Conservation Laws and the Mathematical Theory of Shock Waves, SIAM Regional Conference Series in Applied Mathematics, 11 (1972).

[13] S. OsHER, Riemann solvers, the entropy condition and difference approximations, SIAM J. Numer. Anal., 21 (1984), pp. 217-235.

[14] S. OShER AND S. ChAKRAVARThy, High resolution schemes and entropy condition, SIAM J. Numer. Anal., 21 (1984), pp. 955-984.

[15] P. L. RoE, Some contributions to the modeling of discontinuous flows, Lect. Notes Appl. Math., 22 (1985), pp. 163-193.

[16] P. K. Sweby, High resolution schemes using flux limiters for hyperbolic conservation laws, SIAM J. Numer. Anal., 21 (1984), pp. 995-1011.

[17] E. TADMOR, Convenient total variation diminishing conditions for nonlinear difference schemes, SIAM J. Numer. Anal., 25 (1988), pp. 1002-1014.

[18] B. VAN LEER, Towards the ultimate conservative difference scheme II. Monotonicity and conservation combined in a second order scheme, J. Comput. Phys., 14 (1974), pp. 361-370.

[19] A. Vol'Pert, The space BV and quasi-linear equations, Math. USSR Sb, 73 (1967), pp. 255302.

[20] H. YANG, On Wavewise Entropy Inequalities for High-Resolution Schemes I: The Semi-discrete case, Math. Comp., 65 (1996), pp. 45-67.

[21] H. YANG, On Wavewise Entropy Inequalities for High Resolution Schemes II: Fully discrete MUSCL Schemes with Exact Evolution In Small time, SIAM. J. Numer. Anal., 36:1 (1999), pp. $1-31$.

[22] H. Yang and N. Jiang, On Wavewise Entropy Inequalities for High-Resolution Schemes with source terms I: The Semi-discrete case, Methods and Applications of Analysis, 10:4 (2003), pp. 487-512. 
N. JIANG 